Article

\title{
Do the Mega and Titan Tests Yield Accurate Results? An Investigation into Two Experimental Intelligence Tests
}

\author{
David Redvaldsen \\ Sociology and Social Work, University of Agder, 4604 Kristiansand, Norway; david.redvaldsen@uia.no
}

Received: 18 October 2019; Accepted: 28 April 2020; Published: 29 April 2020

check for updates

\begin{abstract}
The Mega and Titan Tests were designed by Ronald K. Hoeflin to make fine distinctions in the intellectual stratosphere. The Mega Test purported to measure above-average adult IQ up to and including scores with a rarity of one in a million of the general population. The Titan Test was billed as being even more difficult than the Mega Test. In this article, these claims are subjected to scrutiny. Both tests are renormed using the normal curve of distribution. It is found that the Mega Test has a higher ceiling and a lower floor than the Titan Test. While the Mega Test may thus seem preferable as a psychometric instrument, it is somewhat marred by a number of easy items in its verbal section. Although official scores reported to test-takers are too high, it is likely that the Mega Test does stretch to the one in a million level. The Titan Test does not. Testees who had previously taken standard intelligence tests achieved average scores of 135-145 IQ on those. Since the mean of all scores on the Mega and Titan Tests was found to be IQ 137 and IQ 138, respectively, testees had considerable scope to find their true level without ceiling effects. Both are unusual and non-standard tests which require a great deal of effort to complete. Nevertheless, they deserve consideration as they represent an inventive experimental method of measuring the very highest levels of human intelligence and have been taken by enough subjects to allow norming.
\end{abstract}

Keywords: intelligence; Gaussian distribution; giftedness; psychometrics; genius

\section{Introduction}

Intelligence tests were invented by Alfred Binet and his student Théodore Simon in 1905 with the purpose of identifying pupils in need of remedial help in French public education. Within a few years, they had been translated into English and were to reach their apogee in the United States where Lewis M. Terman, a young professor of education at Stanford University, made his reputation as the foremost authority on all matters connected with intelligence. Terman's first book on the topic, The Measurement of Intelligence, featured examples of individuals within the various classifications [1]. By the time his The Intelligence of School Children was published three years later, it was clear that Terman's primary interest was in subjects scoring at the highest levels [2]. He had already begun a study of exceptional children, which became the basis for longitudinal research into the lives and careers of the gifted.

This study, published in five volumes as Genetic Studies of Genius between 1926 and 1959, required the construction of a special instrument to accommodate Terman's subjects as adults, called the Concept Mastery Test [3]. This marked the beginning of experimental research on adults in the intellectual stratosphere using psychological techniques. Due to the rarity of the individuals concerned, it was fraught with practical difficulties. One possible method was to give adolescents achievement tests designed for adults. That was the approach chosen by the Study of Mathematically Precocious Youth, which began in 1971 at Johns Hopkins University and which, despite its name, also considered verbal 
ability. Students who scored at the highest levels on College admission tests at the age of 13 must, logically, be even brighter than the most able ordinary freshmen [4].

In contrast to this well-funded academic project, extending the scale of intelligence to the highest conceivable levels was an endeavor solely taken up by amateurs. Their method was to publish self-authored tests and to form societies for those who received the highest scores on them. In this way, more could be learned about intellects of the very highest order. Omni magazine, devoted to popular science and science fiction, published three such tests between 1979 and 1990. Because of Omni's large readership, enough responses were received to allow official scoring of these tests with at least a semblance of being exact. The procedure of the designers was to compare the number of correct answers yielded by participants and their self-reported previous performance on standard educational or intellectual scales. The data were submitted by mail. It was, of course, an experimental method, because there could be no supervision of test-takers or control of whether the reported scores on the standard tests were accurate.

These three tests were the Langdon Adult Intelligence Test, the Mega Test and the Titan Test. They are the only credible tools for the measurement of intelligence at levels above the ceilings of the traditional instrument the Stanford-Binet, first developed by Terman, and the Wechsler Adult Intelligence Scale (WAIS). The Concept Mastery Test is purely verbal or educational, which means it cannot capture numerical or logical thinking, seen as essential components of intelligence in all modern studies [5-7].

\section{Object}

In this paper, we will investigate the Mega and Titan Tests, designed by Ronald K. Hoeflin and published in Omni magazine in April 1985 and April 1990 respectively [8,9]. We wish to discover whether their author's claims for them are well founded. The Mega Test was billed as discriminating up to the one in a million level of the general population as for intelligence, while the Titan Test was designed to be even more difficult. If this is verified, they could potentially help to identify the most gifted adults imaginable. This is a topic of some interest as the study of genius is one of the oldest concerns within psychology [10-12]. As the Mega and Titan Tests are relatively unknown tools serving a niche market, we additionally wish to consider whether they are suitable for wider use by psychologists. The Langdon Adult Intelligence Test would also have been considered if its norming data had been made public [13]. It is believed to have been taken by more than 20,000 individuals and was normed on the basis of recognized intelligence tests [14].

\section{Method}

The designer's method is the experimental measurement of the very highest levels of human intelligence. It is experimental in the sense of being based on unrecognized techniques which are put forward for consideration. It is also experimental in retaining some features of previous practice, while changing others in pursuit of a particular outcome. Dr Hoeflin saw intelligence as a composite of verbal, numerical and spatial skills. Standard item formats such as analogies, number series, logical progression and mental manipulation of three-dimensional objects were included. However, he dispensed with a time limit and permitted the use of reference materials and, in one case, pocket calculators. These novelties may be justified by seeking to privilege intellectual power over speed and the correct application of knowledge rather than merely possessing it. Our method, on the other hand, goes back to first principles in simply mapping the raw scores obtained on the tests onto the normal curve of distribution. An assumption behind the norming of tests of mental ability is that intelligence is a variable characteristic which is distributed normally. This also matches the empirical realities, according to a meta-study of ten mostly well-known surveys of intellect [15]. We are not primarily interested in the predictive validity of the tests since they are designed for adults, but whether they can be used to identify the presence of intellectual power beyond what the standard tests allow. If they 
do, the entire range of human intellect would be available for study according to a common criterion (psychologically tested intelligence).

A critique of the Mega Test already exists which focused on the violation of psychological practices inherent in accepting testing without supervision and norming from self-reported data [16]. The reviewer felt that its accuracy would be increased if the test were taken under controlled conditions. As it stands, the library resources available to test-takers are a factor in the score generated. Even so, Dr Carlson recognized that the author made such a choice for practical reasons. Hoeflin did not have access to a large pool of individuals who could take the test under controlled conditions for norming purposes. It might be added here that allowing reference materials closed off a potential avenue to cheating and that efficiency in using dictionaries, thesauri and encyclopedias in any case probably correlate to intelligence. Therefore, instead of pursuing the available resources angle further, we will examine, using the available data, whether Hoeflin's tests truly identify giftedness beyond what the recognized tests do. If they do not, the rationale behind them disappears however the resources issue is settled.

\section{Limitations}

As we are probing an experimental attempt to extend the range of the scale of intelligence, we are aware that our research has several limitations. Chief among them is the problem of validity intrinsic to the Mega and Titan Tests. No data have been published which shows Titan Test correlations with other intelligence tests, while the Mega Test correlates only 0.374 with the Stanford-Binet and a mere 0.137 with the WAIS [16]. It is true that it correlates more highly with some other intelligence tests $(0.565$ with the Army General Classification Test and 0.562 with Cattell), but can we be sure that these tests measure what they purport to do? The lack of a time limit has the effect of rewarding persistence and intense interest in the subject matter over actual capacity in a real-world setting. In removing speed as a factor, the Mega and Titan Tests also define intelligence differently to the established understanding manifest in virtually all other tests, while simultaneously giving rise to scores on what is presented as the same scale. Most of the questions on these tests are very difficult and risk conflating puzzle-solving skills with general ability. Especially the Titan Test contains too many spatial items to be representative of $g$, the general factor underlying thinking, see [17]. Neither it nor the Mega Test can be used on the general population, and consequently the lowest raw scores are uncertain. The tests are, however, reliable, as they would be scored identically by any marker since there is a single correct answer to each question.

A present limitation for us is that we are relying on a non-standard source (a web page) for scores on the Titan Test, as Omni magazine did not continue coverage of the topic after 1990. We have no direct method of norming the Mega Test other than by the self-reported previous test scores of Omni participants (also from the web page) and the Titan Test, in turn, is normed from self-reported scores on the Mega Test. Although the tests are examined here in case they are, or may be adapted to be, useful to psychologists and researchers, there is no guarantee that the highest scorers on them are representative of the statistical group to which they belong. They are a self-selected sample, possibly with excess time on their hands.

\section{The Mega Test}

The Mega Test consists of 48 items, of which 24 are verbal analogies, 12 spatial problems, 6 number series and 6 other numerical problems. Two of the questions are multiple choice, but there is no penalty for wrong answers on these or other questions. There is no time limit, though it is suggested the subject spend no more than one month. Reference materials and pocket calculators are permitted. Given that thesauri can be used, a number of questions in the verbal section become relatively easy. A facsimile of the original is available to the reader [18].

The January 1986 issue of Omni carried a score report for the magazine's readership who had taken the Mega Test as printed in the April 1985 issue [19]. It was stated that about 3200 readers had submitted answers to Dr Hoeflin, and that the median score was 15. An accompanying graph allowed 
information to be read off about the frequency of each raw score. Because this was given in tens, it required some concentration on our part to arrive at an exact number of readers who had achieved a particular raw score. We are convinced that our reading is accurate, which was confirmed by our grand total of 3258 testees.

The data used for the subsequent calculations are given in Table 1 below.

Table 1. The frequency of raw scores on the Mega Test among Omni readers.

\begin{tabular}{|c|c|c|}
\hline Score & Frequency & Product \\
\hline 0 & 3 & 0 \\
\hline 1 & 20 & 20 \\
\hline 2 & 30 & 60 \\
\hline 3 & 43 & 129 \\
\hline 4 & 77 & 308 \\
\hline 5 & 103 & 515 \\
\hline 6 & 138 & 828 \\
\hline 7 & 140 & 980 \\
\hline 8 & 125 & 1000 \\
\hline 9 & 160 & 1440 \\
\hline 10 & 140 & 1400 \\
\hline 11 & 160 & 1440 \\
\hline 12 & 145 & 1740 \\
\hline 13 & 142 & 1846 \\
\hline 14 & 128 & 1792 \\
\hline 15 & 140 & 2100 \\
\hline 16 & 138 & 2208 \\
\hline 17 & 126 & 2142 \\
\hline 18 & 104 & 1872 \\
\hline 19 & 120 & 2280 \\
\hline 20 & 100 & 2000 \\
\hline 21 & 108 & 2268 \\
\hline 22 & 90 & 1980 \\
\hline 23 & 80 & 1840 \\
\hline 24 & 77 & 1848 \\
\hline 25 & 60 & 1500 \\
\hline 26 & 70 & 1820 \\
\hline 27 & 40 & 1080 \\
\hline 28 & 49 & 1372 \\
\hline 29 & 43 & 1247 \\
\hline 30 & 22 & 660 \\
\hline 31 & 38 & 1178 \\
\hline 32 & 40 & 1280 \\
\hline 33 & 28 & 924 \\
\hline 34 & 11 & 374 \\
\hline 35 & 17 & 595 \\
\hline 36 & 18 & 648 \\
\hline 37 & 10 & 370 \\
\hline 38 & 8 & 304 \\
\hline 39 & 8 & 312 \\
\hline 40 & 3 & 120 \\
\hline 41 & 4 & 164 \\
\hline 42 & 7 & 294 \\
\hline 43 & 3 & 129 \\
\hline 44 & 3 & 132 \\
\hline 45 & 1 & 45 \\
\hline TOTALS & 3258 & 48,899 \\
\hline
\end{tabular}


The mean was thus $48,899 / 3258=15$. The standard deviation was calculated as shown in Table 2 below.

Table 2. Deviation of scores from the mean on the Mega Test.

\begin{tabular}{|c|c|c|c|c|}
\hline Score & Frequency & Deviation & Deviation $^{2}$ & Product \\
\hline 0 & 3 & -15 & 225 & 675 \\
\hline 1 & 20 & -14 & 196 & 3920 \\
\hline 2 & 30 & -13 & 169 & 5070 \\
\hline 3 & 43 & -12 & 144 & 6192 \\
\hline 4 & 77 & -11 & 121 & 9317 \\
\hline 5 & 103 & -10 & 100 & 10,300 \\
\hline 6 & 138 & -9 & 81 & 11,178 \\
\hline 7 & 140 & -8 & 64 & 8960 \\
\hline 8 & 125 & -7 & 49 & 6125 \\
\hline 9 & 160 & -6 & 36 & 5760 \\
\hline 10 & 140 & -5 & 25 & 3500 \\
\hline 11 & 160 & -4 & 16 & 2560 \\
\hline 12 & 145 & -3 & 9 & 1305 \\
\hline 13 & 142 & -2 & 4 & 568 \\
\hline 14 & 128 & -1 & 1 & 128 \\
\hline 15 & 140 & 0 & 0 & 0 \\
\hline 16 & 138 & 1 & 1 & 138 \\
\hline 17 & 126 & 2 & 4 & 504 \\
\hline 18 & 104 & 3 & 9 & 936 \\
\hline 19 & 120 & 4 & 16 & 1920 \\
\hline 20 & 100 & 5 & 25 & 2500 \\
\hline 21 & 108 & 6 & 36 & 3888 \\
\hline 22 & 90 & 7 & 49 & 4410 \\
\hline 23 & 80 & 8 & 64 & 5120 \\
\hline 24 & 77 & 9 & 81 & 6237 \\
\hline 25 & 60 & 10 & 100 & 6000 \\
\hline 26 & 70 & 11 & 121 & 8470 \\
\hline 27 & 40 & 12 & 144 & 5760 \\
\hline 28 & 49 & 13 & 169 & 8281 \\
\hline 29 & 43 & 14 & 196 & 8428 \\
\hline 30 & 22 & 15 & 225 & 4950 \\
\hline 31 & 38 & 16 & 256 & 9728 \\
\hline 32 & 40 & 17 & 289 & 11,560 \\
\hline 33 & 28 & 18 & 324 & 9072 \\
\hline 34 & 11 & 19 & 361 & 3971 \\
\hline 35 & 17 & 20 & 400 & 6800 \\
\hline 36 & 18 & 21 & 441 & 7938 \\
\hline 37 & 10 & 22 & 484 & 4840 \\
\hline 38 & 8 & 23 & 529 & 4232 \\
\hline 39 & 8 & 24 & 576 & 4608 \\
\hline 40 & 3 & 25 & 625 & 1875 \\
\hline 41 & 4 & 26 & 676 & 2704 \\
\hline 42 & 7 & 27 & 729 & 5103 \\
\hline 43 & 3 & 28 & 784 & 2352 \\
\hline 44 & 3 & 29 & 841 & 2523 \\
\hline 45 & 1 & 30 & 900 & 900 \\
\hline TOTALS & 3258 & & & 221,306 \\
\hline
\end{tabular}


The variance can accordingly be calculated as $221,306 / 3258=67.93$. The standard deviation is therefore $\sqrt{ } 67.93$ or 8.24 .

As established, we have a mean of 15 and a standard deviation of 8.24. It was also reported in the January 1986 issue of Omni that the mean IQ for its readers on the Mega Test had been 141 (on the scale used by the Stanford-Binet, which traditionally had a standard deviation of 16). Dr. Hoeflin arrived at this value by collating previous scores on intelligence and achievement tests reported by participants. We have chosen to calculate the mean IQ on the basis of four intelligence tests alone: the Cattell, the California Test of Mental Maturity (CTMM), the WAIS and the Stanford-Binet (see Table 3 below). Scores were reported on other tests too [20], but since the standard deviations for those are not as clear as for our chosen instruments, they were not taken into consideration by us. This is particularly true for the Army General Classification Test.

Table 3. Previous scores on other tests and raw scores on the Mega Test.

\begin{tabular}{|c|c|c|c|c|c|c|c|}
\hline $\begin{array}{l}\text { Test and Its } \\
\text { Standard } \\
\text { Deviation }\end{array}$ & Previous IQs & $\begin{array}{l}\text { Mega Test } \\
\text { Raw Scores }\end{array}$ & $\begin{array}{c}\text { Sum } \\
\text { Previous } \\
\text { IQs }\end{array}$ & $\begin{array}{c}\text { Sum Mega } \\
\text { Test Raw } \\
\text { Scores }\end{array}$ & $\begin{array}{l}\text { Number of } \\
\text { Observations }\end{array}$ & $\begin{array}{c}\text { Mean } \\
\text { Previous } \\
\text { IQ }\end{array}$ & $\begin{array}{c}\text { Mean } \\
\text { Mega Test } \\
\text { Raw Score }\end{array}$ \\
\hline $\begin{array}{c}\text { Cattell } \\
\text { (S.D. 24) }\end{array}$ & $\begin{array}{l}178,155,178,157,156, \\
163,177,157,175,158, \\
161,143,154,140,135, \\
157,152,172,154,161, \\
162,155,159,157,159, \\
150,151,155,159,151, \\
139,148,148,151,160, \\
147,147,139,135,150, \\
137,146,142,130,152, \\
155,142,151,134,140 .\end{array}$ & $\begin{array}{c}44,39,34,33, \\
33,29,28,27, \\
27,26,25,24, \\
24,23,23,21, \\
21,21,20,19, \\
18,16,15,15, \\
15,15,14,13, \\
13,13,12,12, \\
11,10,10,10,9, \\
9,8,7,7,6,5,5, \\
5,5,4,3,3,3 .\end{array}$ & 7634 & 832 & 50 & 152.68 & 16.64 \\
\hline $\begin{array}{c}\text { CTMM } \\
\text { (S.D. 16) }\end{array}$ & $\begin{array}{c}174,148,137,139,153, \\
143,135,132,130,130, \\
136,128,142,138,136, \\
144,105,156,139,148, \\
135,145,153,138,135, \\
148,142,140,144,143, \\
142,145,121,143,123, \\
135,148,135,136,140, \\
132,147,139,135,135, \\
134 .\end{array}$ & $\begin{array}{c}43,33,29,27 \\
26,25,24,24 \\
24,24,24,23 \\
21,21,20,20 \\
19,18,17,16 \\
16,16,15,15 \\
15,15,15,14 \\
13,13,13,12 \\
11,11,11,11 \\
10,9,7,7,7,6 \\
5,5,5,5 .\end{array}$ & 6406 & 760 & 46 & 139.26 & 16.52 \\
\hline $\begin{array}{c}\text { WAIS } \\
\text { (S.D. 15) }\end{array}$ & $\begin{array}{l}133,149,149,140,140 \\
133,134,150,130,137 \\
139,138,144,140,130 \\
130,164,125,115,133 \\
130,152,134,140,138 .\end{array}$ & $\begin{array}{c}33,32,32,30 \\
29,28,28,26 \\
25,24,22,21 \\
20,17,16,16 \\
15,13,10,9,8 \\
8,7,7,5 .\end{array}$ & 3447 & 481 & 25 & 137.88 & 19.24 \\
\hline $\begin{array}{l}\text { Stanford-Binet } \\
\quad \text { (S.D. 16) }\end{array}$ & $\begin{array}{c}137,124,166,149,143, \\
145,167,148,127,145, \\
149,156,169,149,149, \\
127,138,155,150,126, \\
135,150,158,157,122, \\
138,151,149,134,143, \\
160,139,130,148,139, \\
148,138,137 .\end{array}$ & $\begin{array}{c}40,34,32,28 \\
27,26,26,24 \\
24,24,24,22 \\
22,21,20,20 \\
19,19,19,19 \\
17,17,16,15 \\
15,14,13,13 \\
11,11,11,9,8 \\
8,7,7,4,3\end{array}$ & 5495 & 689 & 38 & 144.61 & 18.13 \\
\hline
\end{tabular}

Converting to the Stanford-Binet equivalent scale used by Hoeflin, we arrive at a mean of IQ 135.12 for those who had previously taken the Cattell, IQ 139.26 for previous CTMM-takers, IQ 140.40 for those who reported scores on the WAIS and IQ 144.61 for former Stanford-Binet testees. The mean for each group on the Mega Test was higher than for the Omni respondents in general. The previous scores on the Stanford-Binet were particularly high, with many above the available ceiling for adults. This leads us to believe that a significant proportion of these were yielded in childhood. Since such scores are not applicable to the norming of an adult intelligence test, we decided to discard the Stanford-Binet data. Using the results from the other tests, we calculated an average of 137.8 IQ at a raw score of 17.13. If a raw score of 17 thus represents an IQ of 138 in round numbers, the mean of 
15 on the Mega Test likely represents an IQ of 137, given the rate of growth on this part of the scale (this will be confirmed below). We therefore base our norming on the mean of 15 being equivalent to an IQ of 137.

The major advantage in introducing the deviation IQ was that it should conform to the normal curve of distribution. The number of scores above the mean do show a generally declining tendency. We therefore decided to map raw scores above the mean onto the normal curve. The shape of the normal curve means that scores taper off sharply above 140 IQ. Any score above the mean or below the mean are less common occurrences, but on this test, raw scores below the mean increase in frequency. Therefore, a different method will be used to calculate raw scores below the mean. We divided scores at and above the 137 IQ-level into intervals of 3 IQ points. To substantiate just how rare the very highest scores are supposed to be, we include a column showing the distribution of scores at or above 137 IQ (see Table 4 below). Statistical tables in books seldom give percentiles for scores more than 3 standard deviations above the mean. Since the previous scores of Mega Test participants were taken from Darryl Miyaguchi's website "Uncommonly Difficult IQ Tests" [20] we additionally decided to use the percentiles calculated by him. This led to the following:

Table 4. Relative frequency of particular IQ levels according to the normal curve of distribution.

\begin{tabular}{cccc}
\hline IQ Interval & Percentile Interval & Percentile Increment & Percentage of Total Scores $\geq$ IQ 137 \\
\hline $137-139$ & $99.0-99.3$ & 0.3 & 40.98 \\
\hline $140-142$ & $99.4-99.57$ & 0.17 & 23.22 \\
\hline $143-145$ & $99.64-99.75$ & 0.11 & 15.02 \\
\hline $146-148$ & $99.8-99.87$ & 0.07 & 9.56 \\
\hline $149-151$ & $99.89-99.93$ & 0.04 & 5.46 \\
\hline $152-154$ & $99.94-99.96$ & 0.02 & 2.73 \\
\hline $155-157$ & $99.97-99.982$ & 0.012 & 1.64 \\
\hline $158-160$ & $99.986-99.991$ & 0.005 & 0.68 \\
\hline $161-163$ & $99.993-99.996$ & 0.003 & 0.41 \\
\hline $164-166$ & $99.997-99.9981$ & 0.0011 & 0.15 \\
\hline $167-169$ & $99.9986-99.9992$ & 0.0006 & 0.082 \\
\hline $170-172$ & $99.9994-99.99966$ & 0.00026 & 0.036 \\
\hline $173-175$ & $99.99975-99.99986$ & 0.00011 & 0.015 \\
\hline $176-178$ & $99.9999-99.99995$ & 0.00005 & 0.007 \\
\hline $179-181$ & $99.99996-99.99998$ & 0.00002 & 0.0027 \\
\hline $182-184$ & $99.999985-99.999992$ & 0.000007 & 0.0016 \\
\hline TOTALS & & 0.732147 & 99.9943 \\
\hline
\end{tabular}

There were 1566 testees who scored 15 or higher on the Mega Test among the Omni readership. We place these scores into the various intervals of the grid constructed from the normal curve of distribution, as can be seen in Table 5 .

The highest scorer on the Mega Test among the Omni readership solved 45 correctly. This represents an IQ of 170 or slightly above. There were three subjects who solved 44 correctly and their associated IQs would be 165-170. We decided to assign them to the 167-169 category, as this allows good approximations of 43 and 42 as raw scores in the 164-166 and 161-163 categories respectively. No reader scored above 45 and we simply do not have the data to tell us what IQ levels these raw scores represent. Because we originally believed the Titan Test to be harder for all raw scores, our aim was to extrapolate from that test to Mega raw scores above 45. It will be shown below, however, that the Mega Test is more difficult near the ceiling. 
Table 5. Distribution of testees in the intervals and associated scores on the Mega Test.

\begin{tabular}{ccc}
\hline IQ Interval & Theoretical Frequency & Associated Scores \\
\hline $137-139$ & 641.7 & $15-19$ \\
\hline $140-142$ & 363.6 & $20-23$ \\
\hline $143-145$ & 235.3 & $24-27$ \\
\hline $146-148$ & 149.7 & $28-31$ \\
\hline $149-151$ & 85.6 & $32-33$ \\
\hline $152-154$ & 42.8 & $34-36$ \\
\hline $155-157$ & 25.7 & $37-39$ \\
\hline $158-160$ & 10.7 & $40-41$ \\
\hline $161-163$ & 6.4 & 42 \\
\hline $164-166$ & 2.4 & 43 \\
\hline $167-169$ & 1.3 & 45 \\
\hline $170-172$ & 0.6 & \\
\hline $173-175$ & 0.2 & \\
\hline $176-178$ & 0.1 & \\
\hline $179-181$ & 0.04 & \\
\hline $182-184$ & 0.01 & \\
\hline TOTAL & 1566.15 & \\
\hline
\end{tabular}

On the basis of the present norming, we believe that the Mega Test is indeed able to yield IQs at the one in a million level, a threshold which is attained at a raw score of either 46,47 or 48 . As for the scores below the mean, we calculate them from one standard deviation equaling 16 IQ points. In this way, each question solved correctly up to the mean adds 16/8.24 or 1.94 IQ points to one's score (see Table 6). This gives us:

Table 6. New norming of the Mega Test 2019.

\begin{tabular}{cccccc}
\hline Raw Score & IQ & Raw Score & IQ & Raw Score & IQ \\
\hline 1 & 110 & 17 & 138 & 33 & 151 \\
\hline 2 & 112 & 18 & 139 & 34 & 152 \\
\hline 3 & 114 & 19 & 139 & 35 & 153 \\
\hline 4 & 116 & 20 & 140 & 36 & 154 \\
\hline 5 & 118 & 21 & 140 & 37 & 155 \\
\hline 6 & 120 & 22 & 141 & 38 & 156 \\
\hline 7 & 121 & 23 & 142 & 39 & 157 \\
\hline 8 & 123 & 24 & 143 & 40 & 158 \\
\hline 9 & 125 & 25 & 143 & 41 & 160 \\
\hline 10 & 127 & 26 & 144 & 42 & 163 \\
\hline 11 & 129 & 27 & 145 & 43 & 165 \\
\hline 12 & 131 & 28 & 146 & 44 & 167 \\
\hline 13 & 133 & 29 & 146 & 45 & 170 \\
\hline 14 & 135 & 30 & 147 & 46 & $170+$ \\
\hline 15 & 137 & 31 & 148 & 47 & $170+$ \\
\hline 16 & 138 & 32 & 149 & 48 & $170+$ \\
\hline
\end{tabular}




\section{The Titan Test}

The Titan Test consists of 48 items, of which 24 are verbal analogies, 6 are number series, 17 are spatial problems and 1 is a complicated calculation. It was designed to be more difficult than the Mega Test. Hardly any questions are intuitive and almost all require a substantial amount of effort. There is no multiple choice nor penalty for incorrect answers. Test-taking time is unlimited and could require more than a month, reference materials are allowed but not calculators or computers. A facsimile of the published test is available to the reader [21].

The Titan Test was attempted by 391 Omni readers. This was only a fraction of the number of responses received for the Mega Test, but is nevertheless high for a test of this nature. The scores of the Omni participants were reported to Mr Miyaguchi and appear on his website [20]. They were as may be seen in Table 7 .

Table 7. Scores on the Titan Test by Omni readers.

\begin{tabular}{|c|c|c|}
\hline Raw Score & Frequency of Score & Product \\
\hline 0 & 21 & 0 \\
\hline 1 & 33 & 33 \\
\hline 2 & 27 & 54 \\
\hline 3 & 25 & 75 \\
\hline 4 & 16 & 64 \\
\hline 5 & 25 & 125 \\
\hline 6 & 20 & 120 \\
\hline 7 & 16 & 112 \\
\hline 8 & 13 & 104 \\
\hline 9 & 15 & 135 \\
\hline 10 & 13 & 130 \\
\hline 11 & 11 & 121 \\
\hline 12 & 10 & 120 \\
\hline 13 & 8 & 104 \\
\hline 14 & 10 & 140 \\
\hline 15 & 8 & 120 \\
\hline 16 & 8 & 128 \\
\hline 17 & 9 & 153 \\
\hline 18 & 10 & 180 \\
\hline 19 & 11 & 209 \\
\hline 20 & 2 & 40 \\
\hline 21 & 8 & 168 \\
\hline 22 & 6 & 132 \\
\hline 23 & 9 & 207 \\
\hline 24 & 7 & 168 \\
\hline 25 & 3 & 75 \\
\hline 26 & 7 & 182 \\
\hline 27 & 2 & 54 \\
\hline 28 & 4 & 112 \\
\hline
\end{tabular}


Table 7. Cont.

\begin{tabular}{|c|c|c|}
\hline Raw Score & Frequency of Score & Product \\
\hline 29 & 3 & 87 \\
\hline 30 & 4 & 120 \\
\hline 31 & 4 & 124 \\
\hline 32 & 3 & 96 \\
\hline 33 & 1 & 33 \\
\hline 34 & 2 & 68 \\
\hline 35 & 2 & 70 \\
\hline 36 & 3 & 108 \\
\hline 37 & 0 & 0 \\
\hline 38 & 1 & 38 \\
\hline 39 & 6 & 234 \\
\hline 40 & 2 & 80 \\
\hline 41 & 1 & 41 \\
\hline 42 & 0 & 0 \\
\hline 43 & 0 & 0 \\
\hline 44 & 1 & 44 \\
\hline 45 & 0 & 0 \\
\hline 46 & 0 & 0 \\
\hline 47 & 0 & 0 \\
\hline 48 & 1 & 48 \\
\hline Totals & 391 & 4556 \\
\hline
\end{tabular}

We begin by calculating the mean, which is $4556 / 391=11.65$. Once we also have the standard deviation for the test, we are in a position to begin norming it. This is computed in Table 8 .

Table 8. Finding the variance and standard deviation of the Titan Test.

\begin{tabular}{ccccc}
\hline Raw Score & Frequency & Dev. from Mean & Deviation $^{2}$ & Product $^{2}$ \\
\hline 0 & 21 & -11.65 & 135.72 & 2850.12 \\
\hline 1 & 33 & -10.65 & 113.42 & 3742.86 \\
\hline 2 & 27 & -9.65 & 93.12 & 2514.24 \\
\hline 3 & 25 & -8.65 & 74.82 & 1870.5 \\
\hline 4 & 16 & -7.65 & 58.52 & 936.32 \\
\hline 5 & 25 & -6.65 & 44.22 & 1105.5 \\
\hline 6 & 20 & -5.65 & 31.92 & 638.4 \\
\hline 7 & 16 & -4.65 & 21.62 & 345.92 \\
\hline 8 & 13 & -3.65 & 13.32 & 173.16 \\
\hline 9 & 15 & -2.65 & 7.02 & 105.3 \\
\hline 10 & 13 & -1.65 & 2.72 & 35.36 \\
\hline 11 & 11 & -0.65 & 0.42 & 4.62 \\
\hline 12 & 10 & 0.35 & 0.12 & 1.2 \\
\hline
\end{tabular}


Table 8. Cont.

\begin{tabular}{|c|c|c|c|c|}
\hline Raw Score & Frequency & Dev. from Mean & Deviation $^{2}$ & Product \\
\hline 13 & 8 & 1.35 & 1.82 & 14.56 \\
\hline 14 & 10 & 2.35 & 5.52 & 55.2 \\
\hline 15 & 8 & 3.35 & 11.22 & 89.76 \\
\hline 16 & 8 & 4.35 & 18.92 & 151.36 \\
\hline 17 & 9 & 5.35 & 28.62 & 257.58 \\
\hline 18 & 10 & 6.35 & 40.32 & 403.2 \\
\hline 19 & 11 & 7.35 & 54.02 & 594.22 \\
\hline 20 & 2 & 8.35 & 69.72 & 139.44 \\
\hline 21 & 8 & 9.35 & 87.42 & 699.36 \\
\hline 22 & 6 & 10.35 & 107.12 & 642.72 \\
\hline 23 & 9 & 11.35 & 128.82 & 1159.38 \\
\hline 24 & 7 & 12.35 & 152.52 & 1067.64 \\
\hline 25 & 3 & 13.35 & 178.22 & 534.66 \\
\hline 26 & 7 & 14.35 & 205.92 & 1441.44 \\
\hline 27 & 2 & 15.35 & 235.62 & 471.24 \\
\hline 28 & 4 & 16.35 & 267.32 & 1069.28 \\
\hline 29 & 3 & 17.35 & 301.02 & 903.06 \\
\hline 30 & 4 & 18.35 & 336.72 & 1346.88 \\
\hline 31 & 4 & 19.35 & 374.42 & 1497.68 \\
\hline 32 & 3 & 20.35 & 414.12 & 1242.36 \\
\hline 33 & 1 & 21.35 & 455.82 & 455.82 \\
\hline 34 & 2 & 22.35 & 499.52 & 999.04 \\
\hline 35 & 2 & 23.35 & 545.22 & 1090.44 \\
\hline 36 & 3 & 24.35 & 592.92 & 1778.76 \\
\hline 38 & 1 & 26.35 & 694.32 & 694.32 \\
\hline 39 & 6 & 27.35 & 748.02 & 4488.12 \\
\hline 40 & 2 & 28.35 & 803.72 & 1607.44 \\
\hline 41 & 1 & 29.35 & 861.42 & 861.42 \\
\hline 44 & 1 & 32.35 & 1046.52 & 1046.52 \\
\hline 48 & 1 & 36.45 & 1328.60 & 1328.60 \\
\hline Totals & 391 & & & 42,455 \\
\hline
\end{tabular}

The variance is therefore $42,455 / 391=108.58$. So the standard deviation is $\sqrt{ } 108.58=10.42$. What IQ level does the mean of 11.65 represent? Unlike for the Mega Test, previous test scores of Omni participants are not available. The only usable data we have for norming is a table of paired scores for testees who attempted both the Mega and the Titan Tests from early 1999, several years after the latter was published in Omni [20]. It is reproduced below as Table 9. 
Table 9. Reported scores on the Mega Test by Titan Test-takers.

\begin{tabular}{|c|c|}
\hline Titan Test Score & Mega Test Score \\
\hline 48 & 44 \\
\hline 46 & 43 \\
\hline 45 & 42 \\
\hline 41 & 37 \\
\hline 41 & 35 \\
\hline 40 & 28 \\
\hline 39 & 40 \\
\hline 39 & 39 \\
\hline 39 & 30 \\
\hline 39 & 21 \\
\hline 38 & 41 \\
\hline 38 & 36 \\
\hline 37 & 33 \\
\hline 37 & 30 \\
\hline 36 & 44 \\
\hline 36 & 35 \\
\hline 36 & 33 \\
\hline 35 & 40 \\
\hline 35 & 36 \\
\hline 34 & 29 \\
\hline 32 & 38 \\
\hline 32 & 35 \\
\hline 32 & 25 \\
\hline 31 & 36 \\
\hline 31 & 32 \\
\hline 31 & 25 \\
\hline 30 & 33 \\
\hline 30 & 31 \\
\hline 29 & 27 \\
\hline 28 & 28 \\
\hline 27 & 26 \\
\hline 26 & 27 \\
\hline 26 & 21 \\
\hline 26 & 21 \\
\hline 25 & 34 \\
\hline 25 & 31 \\
\hline 25 & 19 \\
\hline 24 & 23 \\
\hline 24 & 22 \\
\hline 23 & 33 \\
\hline 23 & 29 \\
\hline 23 & 24 \\
\hline 23 & 22 \\
\hline 23 & 14 \\
\hline 22 & 23 \\
\hline 22 & 20 \\
\hline
\end{tabular}


Table 9. Cont.

\begin{tabular}{|c|c|}
\hline Titan Test Score & Mega Test Score \\
\hline 20 & 24 \\
\hline 20 & 22 \\
\hline 19 & 33 \\
\hline 19 & 23 \\
\hline 18 & 30 \\
\hline 18 & 28 \\
\hline 18 & 20 \\
\hline 17 & 29 \\
\hline 17 & 21 \\
\hline 15 & 18 \\
\hline 15 & 15 \\
\hline 14 & 11 \\
\hline 13 & 18 \\
\hline 12 & 25 \\
\hline 12 & 23 \\
\hline 12 & 19 \\
\hline 11 & 15 \\
\hline 11 & 14 \\
\hline 11 & 11 \\
\hline 10 & 15 \\
\hline 9 & 14 \\
\hline 9 & 12 \\
\hline 8 & 20 \\
\hline 8 & 15 \\
\hline 7 & 16 \\
\hline 7 & 9 \\
\hline 6 & 13 \\
\hline 6 & 12 \\
\hline 5 & 12 \\
\hline 5 & 11 \\
\hline 5 & 9 \\
\hline 4 & 24 \\
\hline 4 & 13 \\
\hline 4 & 8 \\
\hline 3 & 12 \\
\hline 3 & 9 \\
\hline 3 & 4 \\
\hline TOTAL 1870 & TOTAL 2042 \\
\hline
\end{tabular}

This indicates that the mean score on the Titan Test was lower than on the Mega Test for participants who took both. Of the 83 participants, 52 achieved a lower raw score on the Titan Test than on the Mega Test. Their mean score was 22.5 on the Titan Test versus 24.6 on the Mega Test. This is considerably higher than the mean of 11.65 on the Titan Test for all Omni participants and not particularly helpful for determining the IQ at the mean. We decided to find the Mega Test scores of Titan Test-takers who were close to the mean of 11.65 by considering scores at 10 to 13 on the latter. Their Mega Test scores were 18, 25, 23, 19, 15, 14, 11 and 15 compared to their Titan Test scores of 13, 12, 12, 12, 11, 11, 11 and 
10 respectively. Their mean Mega Test score was therefore 17.5 and their mean on the Titan Test was 11.5. Now 17.5 on the Mega Test is equivalent to an IQ of 138.5, so if we estimate a raw score of 11 on the Titan Test as being equivalent to an IQ of 138, we have a base for our norming. It is also the same value as Hoeflin calculated in the official norming and he had access to the test-takers' previous IQ scores [20].

As before, we create a grid taking account of the normal curve of distribution's shape for scores of 11 or above. This is reproduced as Table 10.

Table 10. Relative frequency of particular IQ levels according to the normal curve of distribution.

\begin{tabular}{cccc}
\hline IQ Interval & Percentile Interval & Percentile Increment & Percentage of Total Scores $\geq$ 138 IQ \\
\hline $138-140$ & $99.1-99.4$ & 0.3 & 44.98 \\
\hline $141-143$ & $99.5-99.64$ & 0.14 & 20.99 \\
\hline $144-146$ & $99.7-99.8$ & 0.1 & 14.99 \\
\hline $147-149$ & $99.83-99.89$ & 0.06 & 9.0 \\
\hline $150-152$ & $99.91-99.94$ & 0.03 & 4.5 \\
\hline $153-155$ & $99.95-99.97$ & 0.02 & 3.0 \\
\hline $156-158$ & $99.977-99.986$ & 0.009 & 1.35 \\
\hline $159-161$ & $99.989-99.993$ & 0.004 & 0.6 \\
\hline $162-164$ & $99.995-99.997$ & 0.002 & 0.3 \\
\hline $165-167$ & $99.9976-99.9986$ & 0.001 & 0.15 \\
\hline $168-170$ & $99.9989-99.9994$ & 0.0005 & 0.075 \\
\hline $171-173$ & $99.9995-99.99975$ & 0.00025 & 0.037 \\
\hline $174-176$ & $99.99981-99.9999$ & 0.00009 & 0.013 \\
\hline $177-179$ & $99.99993-99-99996$ & 0.00003 & 0.0045 \\
\hline $180-182$ & $99.99997-99.999985$ & 0.000015 & 0.0022 \\
\hline $183-185$ & $99.999989-99.999995$ & 0.000006 & 0.0009 \\
\hline TOTALS & & 0.666891 & 99.9926 \\
\hline
\end{tabular}

There were 167 test-takers who scored 11 or above and thus qualified to be inserted into our grid. We sort the test-takers into categories on the basis of their raw scores, as can be seen in Table 11.

Table 11. Distribution of testees in the intervals and associated scores on the Titan Test.

\begin{tabular}{ccc}
\hline IQ Interval & Theoretical Frequency & Associated Scores \\
\hline $138-140$ & 75.12 & $11-18$ \\
\hline $141-143$ & 35.06 & $19-23$ \\
\hline $144-146$ & 25.04 & $24-28$ \\
\hline $147-149$ & 15.02 & $29-33$ \\
\hline $150-152$ & 7.5 & $34-38$ \\
\hline $153-155$ & 5 & 39 \\
\hline $156-158$ & 2.25 & 40 \\
\hline $159-161$ & 1 & $41-43$ \\
\hline $162-164$ & 0.5 & $44-47$ \\
\hline $165-167$ & 0.25 & 48 \\
\hline $168-170$ & 0.125 & \\
\hline $171-173$ & 0.063 & \\
\hline $174-176$ & 0.023 & \\
\hline $177-179$ & 0.0075 & \\
\hline $180-182$ & 0.0038 & \\
\hline $183-185$ & 0.0015 & \\
\hline TOTAL & 166.9638 \\
\hline
\end{tabular}


The clustering at the bottom is probably a result of there not being enough test-takers to get more precise results. Getting seven more questions right should raise IQ by much more than two points. It is a problem that we have fewer than 400 test-takers, as the mean IQ is based on the Mega Test to which there were more than 3200 responses. The Titan Test appears to be able to discriminate up to the one in a hundred thousand level, but more extravagant claims do not seem well founded. As for the scores which were below the mean, we use the mean and the standard deviation to estimate them, in preference to the normal curve to which this array of scores does not conform. As before, we assign 16 IQ points to one standard deviation of 10.42, counting from IQ 138, which we placed at raw score 11 (see Table 12). Each question answered correctly up to the mean therefore yields 16/10.42 IQ points, or an increment of 1.54 IQ. Hence, we obtain the following norming for the test:

Table 12. New norming of the Titan Test 2019.

\begin{tabular}{cccccc}
\hline Raw Score & IQ & Raw Score & IQ & Raw Score & IQ \\
\hline 1 & 123 & 17 & 140 & 33 & 149 \\
\hline 2 & 124 & 18 & 140 & 34 & 150 \\
\hline 3 & 126 & 19 & 141 & 35 & 150 \\
\hline 4 & 127 & 20 & 141 & 36 & 151 \\
\hline 5 & 129 & 21 & 142 & 37 & 151 \\
\hline 6 & 130 & 22 & 142 & 38 & 152 \\
\hline 7 & 132 & 23 & 143 & 39 & 154 \\
\hline 8 & 133 & 24 & 144 & 40 & 157 \\
\hline 9 & 135 & 25 & 144 & 41 & 159 \\
\hline 10 & 136 & 26 & 145 & 42 & 160 \\
\hline 11 & 138 & 27 & 145 & 43 & 161 \\
\hline 12 & 138 & 28 & 146 & 44 & 163 \\
\hline 13 & 138 & 29 & 147 & 45 & 165 \\
\hline 14 & 139 & 30 & 147 & 46 & 166 \\
\hline 15 & 139 & 31 & 148 & 47 & 167 \\
\hline 16 & 139 & 32 & 148 & 48 & $168+$ \\
\hline
\end{tabular}

This norming is surprisingly low in context, as virtually all the questions on the Titan Test are difficult, unlike the Mega Test which also includes relatively easy items.

As shown above, the mean of the Mega Test is 15 and the standard deviation is 8.24. For the Titan Test, we found a mean of 11.65 and a standard deviation of 10.42. Each test has 48 items of equal weighting. Let us ascertain theoretically which test is the more difficult at the highest raw score possible.

The z-score for the Mega Test would be $48-15 / 8.24=4.004$

The z-score for the Titan Test would be $48-11.65 / 10.42=3.4884$

Empirical evidence also lends credence to the result that the Mega Test is harder at the highest levels. Out of the 3258 test-takers, there were 21 who scored 40 or higher on the Mega Test, which equals a proportion of $0.64 \%$. For the 391 Titan Test subjects, there were 5 who scored 40 or higher, a proportion of $1.28 \%$. For the paired scores, we notice that the highest scorers on the Titan Test normally achieved a lower raw score on the Mega Test. A perfect score of 48 on the Titan Test, achieved by one subject, equals about 44 on the Mega Test. The second highest score on the Titan Test, when considering only Omni participants, was 44, which equals about 42 on the Mega Test. 


\section{Conclusions}

The renorming of these tests has indicated that the official scores reported to participants are too generous in almost all instances see [20] for a comparison. According to our results, the designer's most recent norming of the Mega Test is too high by six IQ points at a raw score of 10, five IQ points at a raw score of 20, ten IQ points at a raw score of 30 and eleven IQ points at a raw score of 40 . The Titan Test has only been normed once. That norming, we believe, is too high by three IQ points at a raw score of 15, by five IQ points at a raw score of 20, ten IQ points at a raw score of 30 and thirteen IQ points at a raw score of 40. Scores on the Mega Test are boosted because its verbal section contains a number of items which can be solved without much effort (see Appendix A). The verbal section of the Titan Test is more abstruse, requiring greater knowledge, more elaborate fact-finding and more thought as to what is being asked for. Therefore, our norming is almost identical to Dr Hoeflin's up to a raw score of 11.

It is a surprise that the ceiling of the Mega Test seems to be higher than that of the Titan Test. Even a cursory glance at the two tests gives the impression that the Titan Test is harder, and it was designed to be so. There are two possible explanations. The first is that it attracted a more select group of testees. Only 391 Omni readers took the Titan Test, as opposed to more than 3200 for the Mega Test. It is possible that with a larger pool of subjects, the mean would have dropped significantly, which would have pushed the highest scores up. IQs yielded on both the tests relate greatly to the scores of other participants. The second explanation would be that taking the Titan Test involves answering questions which are rather similar. For instance, there are five variations on a single theme in the "probabilities" section (see Appendix B). Solving one of these problems correctly might have made it significantly easier to solve others in the same section. The combination of generally difficult questions with clustering occurring inside sections, may then have led to greater dispersal. The concomitant higher standard deviation would, in that case, have pushed the ceiling down. Mega Test questions tend to be more unlike one another. If we discount the defect associated with its verbal section, we believe it does measure mental ability up to the one in a million mark. (Even if the mean on the test represents an IQ of 131, six points lower than assumed, a raw score of 45 would generate an IQ of at least 167 . A raw score of 48 , not yet achieved by a test taker, would then probably still hit the one in a million level, which is an IQ of 176.) The Titan Test measures up to the one in a hundred thousand mark and, as discussed above, has no defect in its verbal section.

The decisive issue is whether these tests can be useful to psychologists. Our norming does indicate that the tests go above the ceilings of established tests. Subjects who achieve a raw score above 40 are of such exceptional ability that standard tests are unable to measure them adequately. Scores above 5 or so on the Titan Test and scores above 11 or so on the Mega Test also betoken giftedness in the subject. For detecting this, the experimental tests are alternatives to the many accepted tests which operate with a ceiling of only 2 to 2.5 standard deviations above the mean. If the experimental tests were to be adopted by a researcher with the resources necessary to combine them in such a way that the easy verbal and any other faulty items were eliminated, they might serve as a useful complement to other high-range instruments such as the Concept Mastery Test or the Miller Analogies Test [22]. This is especially true because the experimental tests offer many non-verbal questions. New norms would of course have to established for the improved test or tests. Short forms of the tests could also be created which select the best items. Item Response Theory would be useful here. The object would be to choose the items which act as the greatest indicators for the levels of ability which surpass the norms on the standard tests available. The Mega and Titan Tests, however, cannot be used on their own and in their current form by psychologists, owing to the lack of supervision associated with them and the extremely lengthy test procedure.

Funding: This research received no external funding.

Acknowledgments: The author is grateful for the input of the three referees for this journal any remaining deficiencies are entirely his own.

Conflicts of Interest: The author declares no conflict of interest. 


\section{Appendix A. Examples of Items from the Verbal Section of the Mega Test}

1. NIGHT is to DAY as NOCTURNAL is to?

2. HEEL is to ACHILLES as BOX is to?

3. SHOE is to COBBLER as BARREL is to?

5. $\frac{1}{2}$ is to SEMI as $1 \frac{1}{2}$ is to?

6. BILLION is to BILLIONTH as GIGA- is to?

11. WATER is to AQUEOUS as SNOW is to?

12. SEA is to LITTORAL as RIVER is to?

\section{Appendix B. Items from the "Probabilities" Section of the Titan Test}

For the following five problems, imagine that there is an ant at each vertex and that the ants all simultaneously crawl along an edge to the next vertex, each ant choosing its path randomly. What is the probability that no ant will encounter each other, either en route or at the next vertex, for each of the following regular polyhedrons?

38. A tetrahedron

39. A cube

40. An octahedron

41. A dodecahedron

42. An icosahedron

\section{References}

1. Terman, L.M. The Measurement of Intelligence: An Explanation of and a Complete Guide for the Use of the Stanford Revision and Extension of the Binet-Simon Intelligence Scale; Houghton Mifflin: Boston, MA, USA, 1916.

2. Terman, L.M. The Intelligence of School Children. How Children Differ in Ability, the Use of Mental Tests in School Grading and the Proper Education of Exceptional Children; Houghton Mifflin: Boston, MA, USA, 1919.

3. Terman, L.M. The Gifted Child Grows Up. Twenty-Five Years' Follow-Up of a Superior Group; Genetic Studies of Genius; Stanford University Press: Stanford, CA, USA, 1947; Volume 4.

4. Lubinski, D.; Benbow, C.P. Study of mathematically precocious youth after 35 years. Uncovering antecedents for the development of math-science expertise. Perspect. Psychol. Sci. 2006, 1, 316-345. [CrossRef] [PubMed]

5. Sternberg, R.J. A Triarchic Mind: A New Theory of Human Intelligence; Viking: New York, NY, USA, 1988.

6. Kline, P. Intelligence. The Psychometric View; Routledge: London, UK, 1990.

7. Jensen, A.R. The g Factor: The Science of Mental Ability; Praeger: Westport, CT, USA, 1998.

8. Morris, S. The world's hardest IQ test. Omni 1985, 7, 128-129, 132.

9. Morris, S. Games. Omni 1990, 12, 90-91.

10. Galton, F. Hereditary Genius: An Enquiry into Its Laws and Consequences; Macmillan: London, UK, 1869.

11. Lombroso, C. The Man of Genius; Scott: London, UK, 1891.

12. Ellis, H. A Study of British Genius; Hurst \& Blackett: London, UK, 1904.

13. Langdon, K. Hardest IQ Test. Omni 1979, 1, 116-120.

14. Morris, S. Brainbuster: Omni's new IQ test. Omni 1981, 3, 96-98.

15. Warne, R.T.; Godwin, L.R.; Smith, K.S. Are there more gifted people than would be expected in a normal distribution? An investigation of the overabundance hypothesis. J. Adv. Acad. 2013, 24, 224-241. [CrossRef]

16. Carlson, R.D. The Mega Test. In Test Critiques; Keyser, D.J., Sweetland, R., Eds.; Pro-Ed: Austin, TX, USA, 1991; Volume 8.

17. Spearman, C. General intelligence objectively determined and measured. Am. J. Psychol. 1904, 15, $201-293$. [CrossRef]

18. Available online: http://www.williamflew.com/omni79d.html (accessed on 25 October 2019).

19. Morris, S. Games. Omni 1986, 8, 112-114.

20. Miyaguchi, D. Uncommonly Difficult IQ Tests. Available online: www.miyaguchi.4sigma.org (accessed on 25 October 2019). 
21. Available online: http://www.williamflew.com/omni139e.html (accessed on 25 October 2019).

22. Zahler, K.A. McGraw-Hill's MAT. Miller Analogies Test; McGraw-Hill: New York, NY, USA, 2006.

(C) 2020 by the author. Licensee MDPI, Basel, Switzerland. This article is an open access article distributed under the terms and conditions of the Creative Commons Attribution (CC BY) license (http://creativecommons.org/licenses/by/4.0/). 